\title{
Research on the Progress of Computer Artificial Intelligence Algorithm
}

\author{
Chao Wang \\ Chongqing Business Vocational College, Chongqing, 410331, China
}

Keywords: computer, computer artificial intelligence algorithm,progress.

\begin{abstract}
At present, computer artificial intelligence algorithm is still in development, and its application stability and accuracy is not very good. Therefore, relevant researchers are required to be committed to the research on computer artificial intelligence algorithm in order to gradually improve the level of computer artificial intelligence algorithm. Based on this, this paper will take computer artificial intelligence algorithm as the research object to make a research on the progress of computer artificial intelligence algorithm.
\end{abstract}

\section{Introduction}

Artificial intelligence is a branch of computer science,and its reasonable application can imitate the thinking activities of human brain such as reasoning, learning, thinking and planning so as to effectively solve more complicated and cumbersome matters such as medical diagnosis, weather forecast, transportation dispatching and management decision. Seen from the current practical situation of computer technology application, people have raised increasingly higher requirements for computer application. In order to meet the application requirements of people, it is very necessary to develop and apply computer artificial intelligence algorithm since it can solve more complicated problems or matters to simplify people's production and life ${ }^{[1]}$. In view of this, it is very necessary to strengthen the research on computer human intelligence algorithm.

\section{Artificial Intelligence}

(A) Definition of artificial intelligence

Professor Nelson from the United States defines artificial intelligence as follows: "Artificial intelligence is a discipline about knowledge, that is, a science about how to express, acquire and use knowledge." This definition of artificial intelligence is relatively extreme. Based on his understanding of artificial intelligence, Professor Winston from China thinks artificial intelligence studies how to make computer do the intelligent work which can be done by people only. Combined with the summaries made by many professors and scholars, the academic circle simply defines artificial intelligence as an emerging technology science which integrates theory, method, technology and application system and is mainly used for the research and development of the intelligence used to simulate humans. Based on the above summaries, it can be determined that the essence of artificial intelligence is that human studies an ability to simulate human thinking activities, aiming at making an in-depth scientific research on artificial intelligence in order to enhance the function of artificial intelligence and extend artificial intelligence to more fields.

(B) Overview of artificial intelligence theory

Artificial intelligence is a branch of computer science, and its research involves a lot of disciplines including computer science, psychology, philosophy, linguistics, etc. Precisely because of the support ofvarious disciplines, artificial intelligence can simulate the thinking activities ofhuman brain such as reasoning, learning, thinking, and planning on the basis of computer in order to solve more complicated and cumbersome matters such as medical diagnosis, weather forecast, and transportation dispatching. Of course, the key to realizing the effective application of artificial intelligence lies in the setting of computer programs, that is, the research object to be represented by symbols uses the 
heuristic reasoning method to conduct the heuristic reasoning of the research object, and strengthen the structure control of the research object, thus ensuring the accuracy and effectiveness of reasoning results $^{[2]}$.

(C) Knowledge-based system

\section{Overview of knowledge system application}

Logical reasoning system, as one of knowledge systems, is characterized by rationality, completeness and easy processing, which supports computer artificial intelligence algorithm. The reason why the logical reasoning system can get effective application is that the system needs rationality to ensure that a derived conclusion is "true"; it needs completeness to ensure that the reasoning will eventually lead to a true conclusion; it needs easy processing to ensure the feasibility of the conclusion. Based on the above logical relationship, a well-formed formula can be combined. So in the process of logical reasoning, it can be said that the conclusion is true or feasible only after it is demonstrated that the well-formed formula is set up.

\section{Relevant knowledge of artificial intelligence system}

The high-level operation of an intelligent program requires the relevant factual knowledge, rule knowledge, control knowledge and meta-knowledge.

The so-called factual knowledge refers to the knowledge of some things in the problem environment such as classification of things, attribute of things, scientific facts related to things, objective facts of things, etc. Therefore, things can be made public and considered to be public knowledge.

The so-called rule knowledge refers to the casual knowledge related to the moral behaviors and actions of things, which is dynamic. Seen from the application of rule knowledge, heuristic knowledge is applied more frequently since it can assist with the research on artificial intelligence.

Control knowledge refers to the solving steps and technical knowledge of relevant problems, including which action should be chosen for execution when a variety of actions are activated. Expert system is one of the main research directions of artificial intelligence, and in the research process of expert system, control knowledge is used to explain the execution of an action ${ }^{[3]}$.

\section{Knowledge and its representation}

Knowledge can be described from some aspects (including scope, purpose, and effectiveness). The scope of knowledge is from specific to general, the purpose of knowledge is from description to designation, and the effectiveness of knowledge is from determinate to uncertain. Based on this, knowledge will be considered as a general method to study whether a machine can express the feasibility and effectiveness of knowledge, so a description can be made of a certain or a group of knowledge to explain some human behaviors or work in some fields, thus effectively solving problems or work.

\section{Review of Artificial Intelligence Research Stages}

Reviewing the development history of artificial intelligence, it can be determined that the research and development of artificial intelligence has exhausted the energy of a large number of researchers. Artificial intelligence was put forward in the 1950s for the first time, and after that, scientists were aware of the importance of artificial intelligence, and then committed to the research on the machine of artificial intelligence, hoping that the machine can imitate most human thinking activities and replace human brain work, which was realized in the period when the fifth-generation computer appeared. On the whole, the development of artificial intelligence has experienced the following stages:

The first stage is the rise and fall of artificial intelligence. 
The concept of artificial intelligence and relevant research results introduced in the 1950s demonstrated to a large extent that the application of artificial intelligence could help people do some labor work to make people's production and life easier and simpler. At that time, people were quite interested in the research on artificial intelligence because of the support by the results and relevant data of artificial intelligence. But good times didn't last long. Artificial intelligence was not widely applied and developed as expected, but was gradually neglected and forgotten due to the limited technical level at that time, which made artificial intelligence suffer a great setback.

The second stage is a new climax of artificial intelligence research.

Standing in the moment and looking back on history, the most worth celebrating in the development of artificial intelligence is that artificial intelligence hasn't disappeared after a failure or setback. After a short trough, some scientists didn't give up the research on artificial intelligence, but made a more in-depth research with the support of the original theory and data. Until the 1970s, scientists launched expert system finally that appeared in front of people as the second generation of artificial intelligent, which made people re-recognize the importance and application of artificial intelligence, let scholars and scientists join in the artificial intelligence research, and accelerated the pace that artificial intelligence was involved in people's production and life. The research and development this time pushed artificial intelligence onto a new climax so that more and more people faced up to and paid attention to it, which was demonstrated by the establishment of the International Joint Conference on Artificial Intelligence ${ }^{[4]}$.

The third stage is a great development of artificial intelligence.

After the second stage of development, scientists, scholars and relevant people studied the artificial intelligence system more systematically and professionally. For example, Japan planned and implemented the "Fifth Generation Computer Development" program, aiming at increasing the logical reasoning speed of computer to its numerical computation speed. In this case, the fifth generation computer was launched in the 1980s, laying a foundation for more effective and extensive subsequent application of intelligent computer.

The fourth stage is the second climax of artificial intelligence research.

In the case of the vigorous development of science and technology, the research and application of Internet technologies have been increasingly mature so that a variety of advanced technologies can be used for the artificial intelligence research as an attempt. Precisely because of this attempt, a distributed computer artificial intelligence algorithm was introduced to turn the previous single research object into a group of research objects, which improved the practicality of artificial intelligence, further pushed artificial intelligence to the second research climax, and laid a foundation for people to effectively use artificial intelligence to solve more complicated and cumbersome problems.

\section{Research ofComputer Artificial Intelligence Algorithm}

In view of the above content analysis, it is determined that the research and application of computer artificial intelligence algorithm can imitate people's thinking ability, and then deal with some complicated problems in real life. In order to demonstrate this point, the writer will use the data model of the automatic course arrangement algorithm for research and description.

(A) Definition of set

With teachers and students of a school as an example, a research was made on the data model of automatic course arrangement algorithm to first determine the definition of set as follows:

Teacher set is A, and its attributes include age, staff number, name, course, course evaluation value, etc.

Course set is B, and its attributes include course name, course number, the number of weeks, the number of hours every time, etc. 
Class set is C, and its attributes include class name (department name, grade, major, and class number), the number of students, etc.

Classroom set is D, and its attributes include classroom name, capacity, category (general or multimedia), etc.

(B) A partial description of association relationship

The construction of mathematical model is carried out based on computer artificial intelligence algorithm. At first, we need to make a partial description of association relationship, that is, to determine as follows:

The relationship among the three values of teacher, course, and class is as follows:

$$
X_{i j k}\left\{\begin{array}{ccc}
1 & <i, j, k> & \text { belong to } R \\
0 & <i, j, k> & \text { belong to } \mathrm{R}
\end{array}\right.
$$

The association relationship among course, time, and classroom is determined and represented as follows:

$$
X_{i j k}\left\{\begin{array}{cc}
1 & i \text { Course is arranged in } \mathrm{k} \text { room at } \mathrm{d} \text { time of weekdays } \\
0 & \text { o.w }
\end{array}\right.
$$

\section{Test conditions for feasible solutions}

Based on the above content analysis, the test conditions for feasible solutions are determined as follows:

First, the same teacher cannot teach two courses in the same time period.

Second, the same classroom cannot be occupied by two courses in the same time period.

Third, the same class cannot be arranged with two courses in the same time period.

\section{An analysis of genetic algorithm solutions}

The calculation of experiment results is made based on genetic algorithm, and finally, the date model of automatic course arrangement algorithm is determined to be used for teachers' course arrangement and teaching research. It is not difficult to find out that the mathematical model constructed by use of the automatic course arrangement algorithm is closer to the actual situation of course arrangement and teaching. So it is very easy to understand whether the course arrangement is rational, teaching is in place, and student learning is effective through an effective analysis made by use of mathematical model.

\section{Progress of Computer Artificial Intelligence Algorithm}

How do we turn a lot of information online into our own knowledge, let the computer find the information we need more quickly according to our needs, and make the computer interface more humanized? We are often confused by all of these problems when we surf the Internet, and people often encounter similar problems as above. So how do we effectively solve the above problems? The answer is the development and promotion of artificial intelligence technology.

Seen from the development status of artificial intelligence technology, expert system is one of the important directions for the research and development of artificial intelligence. The expert system introduced based on artificial intelligence technology imitates the thinking activities of human brain such as reasoning, learning, thinking and planning, and its application to various fields of people's production and life can help people solve some more complicated problems. For example, its application in the medical field can assist doctors with medical diagnosis; its application in industrial production can assist the technical personnel with machinery debugging; its application in meteorology can assist the meteorological personnel with weather forecast; its application in the field of transportation can assist the transportation personnel with transportation dispatching, etc. ${ }^{[5]}$ 
Of course, the application of artificial intelligence technology goes far beyond this, and a more in-depth research and innovation of this technology can make it play a greater role. With reference to the research data of artificial intelligence technology at this stage, it can be determined that the research and development direction of artificial intelligence in the future is to imitate human emotions, that is, injecting the emotional ability into the research of intelligent algorithm in order to make artificial intelligence provide humanized services.

\section{Conclusion}

Looking back on the development history of computer artificial intelligence algorithm, and looking forward to the future development of computer artificial intelligence, it can be determined that artificial intelligence will become an important "helper" in people's production and life, the reason for which is closely related to three characteristics of artificial intelligence as follows: "fast": as a branch of the computer science, artificial intelligence makes full use of the advantages of computer technology to let artificial intelligence computation achieve a faster speed, a higher performance, and a faster basic frequency; "extensive": with the support of various advanced technologies, artificial intelligence can be widely used in various fields of production and life to help people solve more complicated and difficult problems; “deep": artificial intelligence has been stridden forward to intelligence, informatization, and advancement. In conclusion, we should actively make a research on artificial intelligence to let it develop more mature. In this way, the utilization rate of artificial intelligence will be improved significantly in people’s production and life.

\section{References}

[1] Chen Wei. On the Optimization and Application of Artificial Neural Network Based on Swarm Intelligence Algorithm. Jiangnan University, 2007.

[2] Li Yongli, Wu Chong, Wang Kunsheng, et al. On the Artificial Intelligence Algorithm for the Optimization of Time Series Data Model. Computer Engineering and Design, 2011, 32 (12): 4190-4193, 4201.

[3] Zhu Yunchang. A Research on the Improved Algorithm of Two Optimization Methods Based on Swarm Intelligent. Wuhan Institute of Engineering, 2008.

[4] Ma Chao. Application and Comparison of QR Code Recognition System Based on Artificial Intelligence Algorithm. Hangzhou Dianzi University, 2013.

[5] Ma Lei and Yang Lian. Application of Artificial Intelligence Algorithm to the Reactive Power Optimization of Electric Power System. Computer Knowledge and Technology, 2010, 06 (24): 6840-6842. 